\title{
1q21.1 microduplication syndrome
}

INSERM

\section{Source}

INSERM. (1999). Orphanet: an online rare disease and orphan drug data base. 1921.1 microduplication syndrome. ORPHA:250994

1q21.1 microduplication syndrome is a rare partial autosomal trisomy/tetrasomy with incomplete penetrance and variable expression characterized by macrocephaly, developmental delay, intellectual disability, psychiatric disturbances (autism spectrum disorder, attention deficit hyperactivity disorder, schizophrenia, mood disorders) and mild facial dysmorphism (high forehead, hypertelorism). Other associated features include congenital heart defects, hypotonia, short stature, scoliosis. 